Écrire

l'histoire

\section{Écrire l'histoire}

Histoire, Littérature, Esthétique

\section{9 | 2019}

\section{L'historien et les langues}

\title{
Le deuil de la poésie
}

Imaginaire historique et imaginaire linguistique chez Mérimée

\section{Xavier Bourdenet}

\section{OpenEdition}

Journals

Édition électronique

URL : https://journals.openedition.org/elh/1973

DOI : $10.4000 /$ elh. 1973

ISSN : 2492-7457

Éditeur

CNRS Éditions

\section{Édition imprimée}

Date de publication : 1 décembre 2019

Pagination : 151-159

ISBN : 978-2-271-12967-3

ISSN : 1967-7499

Référence électronique

Xavier Bourdenet, «Le deuil de la poésie », Écrire l'histoire [En ligne], 19 | 2019, mis en ligne le 01 décembre 2019, consulté le 21 septembre 2021. URL : http://journals.openedition.org/elh/1973; DOI : https://doi.org/10.4000/elh.1973 


\section{Le deuil de la poésie}

\section{Imaginaire historique et imaginaire linguistique chez Mérimée}

La question linguistique traverse toute l'œuvre de Mérimée, qui se déploie sur plusieurs plans culturels : fiction, archéologie, histoire. Partout, elle est dynamisée d'une tension entre proximité et distance, identité et altérité : comment comprendre la langue de l'autre? comment fonder sur elle une connaissance assurée? Surtout, elle est constamment historicisée: d'une part parce que Mérimée se montre attentif à l'évolution des langues, toujours mise en relation avec celle, plus globale, du processus de civilisation; d'autre part parce qu'il cherche à penser une langue propre à l'histoire et à l'historien qui, c'est ce que je voudrais montrer, se saisit dans le deuil d'une langue poétique, conçue comme originelle.

\section{Langues étrangères, langues étranges}

L'écrivain commence sa carrière sous le masque, par des mystifications présentées comme des traductions : le Théâtre de Clara Gazul (1825) serait l'œuvre d'une comédienne espagnole énergique, fille de bohémienne, traduite par un « Joseph L'Estrange », qui dit d'emblée combien la langue chez Mérimée est toujours vectrice d'une étrangeté radicale, d'une altérité mystérieuse. La Guzla (1827) se présente comme la traduction de pseudo «poésies illyriques recueillies dans la Dalmatie, la Bosnie, la Croatie et l'Herzégovine ", " poèmes sans art, production $\mathrm{d}^{\prime}$ un peuple sauvage ${ }^{1}$ ». La question linguistique est alors prise dans un imaginaire de l'ailleurs, tant géographique que temporel. Elle est instrument de décentrement, d'estrangement à soi, à sa culture, à son présent. La traduction, ici fictive, crée un dispositif duel. Elle est moyen savant de donner accès à une poésie, à une langue populaires et encore presque sauvages, qui se confondent avec celles de la légende : Clara Gazul est « arrière-petitefille du tendre Maure Gazul, si fameux dans les vieilles romances espagnoles ${ }^{2}$ ».

L'historien est sans cesse confronté à cette altérité foncière du passé dont la langue est le meilleur symptôme. Dans toutes ses œuvres historiques, Mérimée souligne l'obstacle qu'est la langue (étrangère, morte) des sources qu'il compulse et sur lesquelles il étaye 
son enquête. Faire œuvre d'historien, c'est toujours, peut-on résumer, être confronté à des lisibilités perdues, à une langue qui ne parle plus ou parle mal. Cela tient à ce qui, pour un romantique comme Mérimée, est au fondement même de l'histoire: une perspective relativiste, l'idée d'une différence des temps et des cultures, qui implique évolution des mœurs et donc des signifiés comme des référents de tout signe linguistique. La question est d'autant plus vive que Mérimée aborde des aires linguistiques et culturelles lointaines et/ ou étrangères: 1'Antiquité des Études sur l'histoire romaine (1844), l'Espagne médiévale de $\mathrm{l}^{\prime}$ Histoire de don Pèdre Ier, roi de Castille (1848), la Russie classique des Faux Démétrius (1853), des Cosaques d'autrefois (1865), etc. L'altérité linguistique est (à) l'image de l'altérité temporelle.

Le sens de termes ou expressions clés pour l'enquête historique est perdu ou a tellement évolué que le sens ancien n'est plus perceptible. C'est qu'il est lié à un état des mœurs devenu étranger et qu'on ne peut reconstituer complètement. L'obscurité de ces signifiants sans signifié tient «à notre ignorance des mœurs et conditions particulières à une société déjà si éloignée de nous ${ }^{3}{ }^{3}$. Ainsi des "compagnies blanches", désignant dans l'Histoire de don Pèdre les compagnies d'aventuriers français, menées par Du Guesclin, venues renforcer les troupes d'Henri de Trastamare, qui dispute la couronne de Castille à son frère don Pèdre. Pourquoi «blanches"? Le sens littéral de l'expression s'est perdu. L'historien rapporte trois hypothèses, sans pouvoir trancher: (1) ces aventuriers portaient une soubreveste blanche ; (2) ils étaient "armés à blanc ", c'est-àdire portaient des armures nouvelles en plaques de fer et non plus des hauberts de mailles; (3) ils portaient une croix blanche comme insigne. S'il penche pour la deuxième hypothèse, Mérimée n'a aucun moyen d'exclure les deux autres, sans être d'ailleurs certain qu'il ne faille pas en envisager une quatrième ${ }^{4}$. L'historien se heurte ici à l'implicite propre à tout discours, qui repose sur un partage des références entre locuteur (les chroniqueurs anciens qu'il compulse) et récepteur (le public premier de ces chroniques). Partage qui n'a plus cours du fait de l'évolution des mœurs, en l'occurrence des techniques et des lois de la guerre. Cicéron fournit un exemple paradigmatique de cette occultation du sens :

Tout le monde connaît le fameux passage de Cicéron, De Rep. II, 22, si souvent controversé, et qui a donné lieu à tant de théories différentes sur le système des comices par centuries, introduit par Servius Tullius. Consultez l'excellent résumé des principales opinions, par M. de Golbéry, dans le septième volume de sa traduction de l'Histoire romaine de Niebuhr ${ }^{5}$.

Cas d'école qui oblige l'historien à consacrer plusieurs pages de son Essai sur la guerre sociale à l'organisation desdits comices sans jamais être assuré de ses dires. Dans Don Pèdre, ce sont la «détestable latinité » de certaines sources ou le castillan du XIV siècle qui lui posent problème, et on voit l'historien solliciter l'aide de M. Bofarull, directeur des Archives d'Aragon, ou de M. Achard, archiviste de Vaucluse, pour éclaircir autant que faire se peut, c'est-à-dire mal, le sens de plusieurs expressions ${ }^{6}$. L'histoire devient, littéralement, traduction. Mais traduction 
incomplète, impossible ou incertaine, contrairement à ce qu'on observait dans les fictions mystificatrices du début de sa carrière.

Ce qui est en revanche commun avec l'œuvre de fiction, c'est le dispositif duel ainsi mis en place. Il s'agit, par une posture ou un ethos savants (ceux de l'éditeur-traducteur-annotateur dans le Théâtre de Clara Gazul ou La Guzla ; ceux de l'historien extrêmement attentif à la critique interne et externe de ses sources, aux opérations de véridiction comme à la logique et à la vraisemblance de son propos dans l'œuvre historiographique), de mettre en scène des traces linguistiques qu'on dira populaires, en cela qu'elles étaient largement partagées, qu'elles avaient une force pragmatique immédiate, devenues objets d'une connaissance médiate plus ou moins assurée.

\section{Les âges et les langues de l'humanité : de la poésie à l'histoire}

Plus généralement, ce dispositif traductologique correspond à la manière dont Mérimée se représente la langue de l'histoire. Il fait de cette dernière l'indice d'un état avancé de la civilisation dans une vision plus fantasmatique que proprement historique des "âges » de l'humanité, d'un processus de civilisation articulé à son avant ou son envers sauvage. Le temps spécifique de l'histoire est à ses yeux toujours second. Il vient après des temps poétiques, qui impliquent un tout autre rapport à la langue. La poésie est première, primitive, originelle dans l'histoire de l'humanité. L'historiographie la tue en s'y substituant. Mérimée conçoit en effet la poésie comme expression de temps barbares ou demi-civilisés :

J'aime les chants populaires de tous les pays et de tous les temps, depuis l'Iliade jusqu'à la romance de Malbrouk. À vrai dire, je ne conçois pas, et c'est peut-être une hérésie, je ne conçois guère de poésie que dans un état de demi-civilisation, ou même de barbarie, s'il faut trancher le mot. C'est dans cet heureux état seulement que le poète peut être naïf sans niaiserie, naturel sans trivialité. Il ressemble alors à un charmant enfant qui bégaye des chansons avant de construire une phrase. Il est toujours amusant, parfois sublime; il m'émeut, parce qu'il croit tout le premier les contes qu'il me débite ${ }^{7}$.

À l'inverse, l'histoire est la langue propre aux temps civilisés. Cela remonte à la fondation même du genre :

Hérodote vint qui gâta tout. Ce n'est pas qu'Hérodote ne fût lui-même un grand poète; mais une histoire nationale écrite en prose, avec critique, sans passion, d'après des mémoires étrangers, constatait un progrès immense de la civilisation et annonçait la fin de l'époque poétique ${ }^{8}$.

À l'époque poétique succède l'époque historique - dont le XIX ${ }^{e}$ siècle serait le 
parachèvement ${ }^{9}$. Avec critique, sans passion, d'après des sources authentiques (les «mémoires »): la méthode d'Hérodote est déjà celle de Mérimée, qu'il résume souvent par l'expression de "saine critique». Elle introduit un rapport distancié à la langue en privilégiant les opérateurs logiques, le regard analytique, et en s'interdisant les deux marques essentielles du discours poétique: l'investissement subjectif dans le discours et la métaphore. Là sont les deux traits d'une langue poétique assimilée à une langue archaïque. Cette dernière se trouve à la fois dans la poésie populaire, "sauvage», et dans les mythes, qui constituent en eux-mêmes une «langue » propre aux temps primitifs, dont l'unité de base, le lexique en quelque sorte, est faite de métaphores :

Pour étudier la mythologie, il faut avant tout, je pense, s'appliquer à connaître sa langue; j'appelle ainsi les figures ou les métaphores par lesquelles les hommes, dans un certain état de civilisation traduisent ordinairement leurs idées. Cette langue, très-pauvre assurément, est, suivant toute apparence, naturelle aux hommes encore grossiers et incultes, car on la trouve en usage dans des pays fort éloignés les uns des autres, et elle sert d'organe à des religions fondées sur des croyances trèsdifférentes. C'est ainsi qu'on ne peut lire les cosmogonies antiques sans être frappé des rapports qu'offrent entre eux les différents récits sur l'origine des choses, je ne dis pas quant à la substance de ces récits seulement, mais surtout quant à la manière de représenter les mêmes idées par les mêmes figures. Toutes ces religions de l'antiquité, qu'on appelle cultes de la Nature, font usage des mêmes métaphores, des mêmes allégories. Tantôt elles considèrent la Nature dans son ensemble, tantôt dans ses propriétés particulières, mais toujours elles la représentent par une suite de personnifications procédant les unes des autres ${ }^{10}$.

L'historien mythologue se pense alors exactement sur le modèle du grammairien, capable de reconstituer le lexique et la syntaxe d'une langue pour lui par définition morte. Conscience historique et conscience linguistique vont de pair, la première se moule sur le modèle épistémologique de la seconde. C'est bien pourquoi l'histoire est l'envers de la poésie. Car cette dernière, pour Mérimée, " ne vit que de métaphores » et, surtout, «fleurit toujours avant la rhétorique et la grammaire ${ }^{11} »$. Les poètes d'autrefois sont heureux parce $q u^{\prime}$ " ignorants des règles et des conventions inventées par les rhéteurs ${ }^{12} »$. Ils sont significativement associés par Mérimée aux «illettrés ${ }^{13}$ » et aux enfants (l'in-fans) qui, on l'a vu, "bégaye[nt] des chansons avant de construire une phrase ", pour mieux souligner le caractère spontané et immédiat de la poésie populaire.

La langue de l'historien en est l'exact envers. Elle refuse obstinément la métaphore. C'est déjà sensible dans la langue du Mérimée écrivain, dont la critique a très tôt souligné la " sécheresse », identifiée à un effet d'économie du discours, à un retrait du commentaire narratorial, mais aussi à une quasi-absence de la métaphore. On rappellera, au passage, que les narrateurs mériméens, figures de savants, sont tous des avatars de l'historien, ceci expliquant sans doute cela : ceux de La Vénus d'Ille et de Carmen sont "archéologues", celui de Lokis, 
"professeur de linguistique comparée ", spécialiste donc de la traduction, et singulièrement du jmoude, la langue la plus ancienne de Lituanie. Ce l'est encore plus chez le Mérimée proprement historien: des Études sur l'histoire romaine aux Cosaques d'autrefois, les très rares métaphores qu'on relève sont toutes parfaitement stéréotypées, presque des catachrèses. Elles empruntent au registre naturel, le plus souvent météorologique, traditionnel pour dire le bouleversement politique et historique ${ }^{14}$ : «tempêtes", « orage » ou «feu » de la révolte. Elles sont l'exception, peu visible, qui confirme la règle d'une langue comme évidée de son pouvoir imageant. Abondent en revanche les remarques d'ordre philologique, qui témoignent d'une conscience métalinguistique, là où le poète était dans un en-deçà de la conscience linguistique. La langue de l'historien s'écrit ainsi dans le deuil de la langue poétique, qui, elle, se parle.

\section{«Une archéologie appliquée à la littérature »}

Mais le deuil n'est pas l'oubli. C'est même tout l'inverse. Il est une conscience de la différence des temps, de l'impossible pouvoir résurrectionnel de l'histoire. Dans un passage célèbre de la préface à son Histoire de France, posant son projet de "résurrection de la vie intégrale ", Michelet, évoque les voix du passé, qu'il entend via les archives :

Dans les galeries solitaires des Archives où j'errai vingt années, dans ce profond silence, des murmures cependant venaient à mon oreille. Les souffrances lointaines de tant d'âmes étouffées dans ces vieux âges se plaignaient à voix basse ${ }^{15}$.

La perspective michelétiste repose ainsi sur l'imaginaire d'une communication directe avec le passé, d'une langue commune qui garde sa force pragmatique puisqu'elle est immédiatement comprise. C'est ce qui ne se produit pas chez Mérimée. La langue du passé reste étrangère, lettre morte, signalée telle. Le rôle de l'historien est de rassembler les traces de cette langue populaire et poétique désormais inaudible, sans efficace immédiate, menacée d'effacement. La langue devient au sens propre objet archéologique, vestige dont la compréhension n'est plus tout à fait assurée et qu'il importe de conserver si l'on veut avoir accès à ce que Mérimée, à la suite de Grote, appelle le "mental stock», le «fonds intellectuel» des hommes du passé $^{16}$, et au-delà, peut-être, à quelque chose d'une origine immémoriale commune, qui précède la différenciation des cultures et que la poésie primitive traduit ${ }^{17}$.

Ces traces archéologiques, auxquelles Mérimée, sous sa double casquette d'écrivain et d'historien, se trouve particulièrement sensible, sont essentiellement de trois ordres. D'abord, la langue du peuple, souvent dialectale, essentiellement orale, c'est-à-dire fragile et évanescente. Par exemple celle des paysans lituaniens que le professeur Wittembach, dans Lokis, veut enregistrer, mais qui lui échappe en grande partie: "Mon glossaire jmoude, note-t-il, était très riche ; 
mais le sens d'un certain nombre de mots appris de la bouche de paysans grossiers demeurait encore pour moi enveloppé $\mathrm{d}^{\prime}$ une obscurité relative ${ }^{18}$. Celle encore des deux polissons de La Vénus d'Ille, ce catalan que le narrateur, " archéologue ", ne comprend qu' «à peu près ${ }^{19}$ ».

Ensuite, les proverbes, soit l'un des éléments typiques de la langue populaire dans ce qu'elle a d'imagé et d'ancré dans l'univers familier du quotidien, de l'expérience commune, qu'elle traduit dans une forme à la fois simple et immédiate. C'est essentiellement sous cette dimension qu'est présent dans Carmen le romani, par exemple, cette langue bohémienne que Mérimée, à la suite de George Borrow, fait dériver du sanscrit, conçu comme langue mère, originelle ${ }^{20}$. Le romani proverbial de Carmen s'entend alors comme écho de l'origine. Mais un écho désormais accessible uniquement de manière médiate et savante, par la traduction en note de bas de page : ce dispositif duel du texte bordé de son paratexte savant et explicatif est l'image même du geste et de la conscience archéologiques, qui consacrent non seulement l'évolution, mais la différence des langues. Lokis fournirait un autre bel exemple. La nouvelle s'écrit en effet à l'enseigne d'un proverbe populaire lituanien qui lui sert d'épigraphe et qui, à condition d'être traduit correctement, ce que la fin de la nouvelle montre comme aléatoire, fournit la clé du récit fantastique (l'homme-ours qu'est le comte Szémioth).

Enfin, les poésies populaires - ballades, romances, chansons et autres légendes -, auxquelles est attribuée la capacité de révéler le primitif, de faire entendre " comme le cri de la nature, souvent sauvage et bizarre, mais quelquefois sublime ${ }^{21} »$. C'est pour sauvegarder quelque chose de cette langue naturelle que Mérimée appelle de ses vœux une «archéologie [...] appliquée à la littérature $^{22}{ }^{2}$. C'est ce qu'il loue dans les entreprises de Marino Vreto (Contes et Poèmes de la Grèce moderne, 1855), de Claude Fauriel (Histoire de la poésie provençale, 1846) ou de Vasile Alexandri (Ballades et Chants populaires de la Roumanie, 1855). Tout comme il salue le projet (resté inabouti) du ministre de l'Instruction publique, Hippolyte Fortoul, d'une vaste enquête visant à recueillir et publier "une collection des anciennes chansons françaises ${ }^{23}$ ». " Je voudrais, précise Mérimée, que l'on conservât les restes de la poésie populaire, comme on conserve les ruines d'un temple dont on a chassé le dieu ${ }^{24}$. " Si la force pragmatique de ces poésies (le dieu du temple), l'acte communicationnel qu'elles permettaient et le lien collectif qu'elles représentaient ont disparu, on doit néanmoins les recueillir comme signes linguistiques dessinant un lieu vide: celui d'une langue naturelle parce que reconnectant l'individu avec l'origine.

Cette archéologie linguistique est partout présente chez Mérimée. Il l'a d'abord singée par la mystification de La Guzla (1827), recueil de "poèmes sans art, production d'un peuple sau$\operatorname{vage}^{25} \gg$. Devenu inspecteur général des Monuments historiques, il a collecté d'authentiques ballades: de sa tournée de 1839, il rapporte plusieurs "poésies populaires corses ", publiées à la fin de ses Notes d'un voyage en Corse (1840), «lamentation[s] funèbre[s] » pour l'essentiel, largement improvisées et qui appellent à la vengeance. Elles sont aux yeux de l'archéologue l'exemple même de ce par quoi "la Corse est encore loin de ressembler au continent ${ }^{26} "$, 
c'est-à-dire encore sauvage. Colomba, la voceratrice, sera bientôt, dans la fiction, la voix même de cette poésie sauvage. Elle dira de la ballata qu'elle improvise qu'elle vient des « aïeux » et qu'il faut la respecter " comme un usage antique ${ }^{27}$ ». Pour que cette langue archaïque garde sa valeur pragmatique, il lui faut des conditions de profération spécifiques, destinées non à des touristes en quête de dépaysement ou de couleur locale, mais à une communauté pour laquelle elle a une fonction sociale. À son frère Orso, civilisé par son passage sur le continent et sa carrière militaire et qui ironise sur cet usage antique de la ballata ( Crois-tu que Charles-Baptiste ne trouvera pas son chemin dans l'autre monde si l'on ne chante de mauvais vers sur sa bière?»), Colomba rétorque: "Souvenez-vous qu'à Ajaccio vous m'avez dit d'improviser pour amuser cette demoiselle anglaise qui se moque de nos vieux usages. Ne pourrai-je donc improviser aujourd'hui pour de pauvres gens qui m'en sauront gré, et que cela aidera à supporter leur chagrin ${ }^{28}$ ? » C'est le privilège de la fiction, chez Mérimée, que de recréer, au moins fugacement et imparfaitement, le cadre pragmatique permettant à la langue poétique, sauvage, de fonctionner, de retrouver sa valeur. L'œuvre de l'historien, elle, n'a pas cette possibilité. Elle n'en assure que plus consciemment sa dimension archéologique en enregistrant constamment les légendes et autres ballades populaires relatives aux personnages historiques qu'elle prend pour objet. Pas une œuvre du Mérimée historien qui ne consigne ces légendes, de don Pèdre ensorcelé par sa maîtresse, une Maria Padilla transformée par le «roman populaire» en magicienne, au faux Démétrius révélant son identité à un noble polonais dans un récit qui « répète à la manière homérique le dialogue de ses héros ${ }^{29}$ ", que les annalistes n'ont pas pu connaître, en passant par les ballades cosaques qui, dans Stenka Razine, doublent constamment la voix de l'historien comme son répondant poétique à nette valeur héroïsante. C'est toujours l'occasion pour l'historien de distinguer sa voix, et sa langue, de celle de la légende, dont les traits sont le recours au merveilleux, l'hypotypose liée à l'énumération des détails et toutes les formes de discours rapporté, singulièrement le discours intérieur: toutes choses impossibles à l'historien, qui écrit entre l'interdit et la nostalgie de cette langue poétique.
D'une certaine manière, l'œuvre de Mérimée est ainsi toujours bilingue, du fait même de l'imaginaire archaïque qui l'habite ${ }^{30}$, tous genres confondus. Elle ne cesse de constater, parfois tente de combler, le hiatus entre une langue poétique fantasmée comme populaire et originelle, comme langue de l'origine, toujours plus ou moins morte, et une langue savante, analytique, qui propose l'archéologie de la première mais qui, dans ce geste analytique même, ne peut que la trahir : au mieux la faire entendre comme écho assourdi. 


\section{Notes}

1 La Guzla, édit. Antonia Fonyi, Kimé, 1994, p. 21.

2 Théâtre de Clara Gazul. Romans et nouvelles, édit. Jean Mallion et Pierre Salomon, Gallimard (Bibliothèque de la Pléiade), 1978, p. 4. [Désormais Pl.].

3 La Conjuration de Catilina, Les Belles Lettres, 2000, p. 40.

4 Histoire de don Pèdre Ier, roi de Castille, Paris, Charpentier, 1848, p. 358, n. 2.

5 Essai sur la guerre sociale, Paris, V. Magen, 1844, p. 353, n. 1.

6 Histoire de don Pèdre Ir ..., p. 280, n. 2, et p. 436, note.

7 «Ballades et Chants populaires de la Roumanie, recueillis et traduits par V. Alexandri, Paris, 1855 ", Moniteur universel, 17 janv. 1856, recueilli dans "Inédits de Mérimée », $R S H$, $\mathrm{n}^{\circ}$ 270, Antonia FonYI (dir.), Mérimée, écrivain, 2003, p. 182-183.

8 Ibid., p. 184.

9 «Nous n'avons plus de ces grandes passions, ni même ces modes tyranniques, comme on en avait autrefois, qui plient tout à un certain caprice et à de certaines conventions. Accoutumés au scepticisme, blasés, indifférents pour le présent, nous pouvons juger plus sainement du passé. [...] Le meilleur temps pour traduire, pour comprendre ceux qui ont inventé, c'est peut-être le temps où l'on n'invente plus ; c'est le nôtre. En résumé, nos progrès, nos qualités, nos défauts même, favorisent aujourd'hui les études historiques. » ( "De l'histoire ancienne de la Grèce ", Mélanges historiques et littéraires [1855], Paris, C. Lévy, 1883 , p. 113).

$10 \mathrm{Ibid} .$, p. 124-125.

11 «Des mythes primitifs. Le Peuple primitif, sa religion, son histoire et sa civilisation, par F. de Rougemont, 1855 », Revue contemporaine, 15 oct. 1855, recueilli dans «Inédits de Mérimée», p. 176-177.

12 «Ballades et Chants populaires de la Roumanie... ", p. 185.
13 Les Contes et Poèmes de la Grèce moderne rassemblés par Marino Vreto sont ainsi «de petits poèmes improvisés pour la plupart par des gens illettrés, mais pleins d'imagination et de verve originale » ("Contes et Poèmes de la Grèce moderne, Paris, E. Audois, 1855 », recueilli dans «Inédits de Mérimée », p. 162). Soit exactement ce que Mérimée peint dans $L a$ Guzla avec le barde fictif Hyacinthe Maglanovich, fils de cordonnier, sans éducation, qui «ne sait ni lire ni écrire » (La Guzla, p. 23) : poésie orale, définie contre la langue écrite.

14 Voir Olivier Ritz, Les Métaphores naturelles dans le débat sur la Révolution, Classiques Garnier, 2016.

15 Jules Michelet, Histoire de France, Paris, A. Lacroix, 1880, t. I, préface de 1869, p. xxxvI.

16 « De l'histoire ancienne de la Grèce », p. 120.

17 Voir le passage cité supra sur les mythes, " récits sur l'origine des choses » qui forment une langue commune à des pays et des cultures éloignés les uns des autres.

18 Lokis, dans Pl., p. 1084.

19 La Vénus d'Ille, dans Pl., p. 736.

20 George Borrow, The Zincali, or an account of the Gypsies of Spain, Londres, 1841. Voir Sarah AL-MATARY, « L'archéologie verbale de Prosper Mérimée : du mythe personnel au mythe scientifique », dans Sarga Moussa (dir.), Le Mythe des bohémiens dans la littérature et dans les arts en Europe, L'Harmattan, 2008, p. 127-147.

21 «Histoire de la poésie provençale par feu M. Fauriel. Cours professéà la Faculté des Lettres en 1832 et 1833, Labille, 1846 », Le Constitutionnel, 17 févr. 1846, recueilli dans «Inédits de Mérimée », p. 155. Sur la vogue et les enjeux de cette poésie populaire romantique, voir Claude Millet, Le Légendaire au XIX siècle. Poésie, mythe et vérité, PUF, 1997.

22 «Contes et Poèmes de la Grèce moderne », p. 162.

23 Ibid.

24 Ibid.

25 La Guzla, p. 21. 
26 Notes d'un voyage en Corse, édit. Pierre-Marie Auzas, A. Biro, 1989, p. 81.

27 Colomba, dans Pl., p. 824.

28 Ibid., p. 825.
29 Épisode de l'histoire de Russie. Les faux Démétrius, édit. Pierre Gonneau, H. Champion, 2012, p. 94.

30 Voir Antonia FonYI, « La passion pour l'archè », dans ID. (dir.), Prosper Mérimée, écrivain, archéologue, historien, Genève, Droz, 1999, p. 197-207. 